\title{
Moving towards solutions to some enduring controversies in visual search
}

\author{
Jeremy M. Wolfe \\ Center for Ophthalmic Research, Brigham and Women's Hospital, 221 Longwood Ave, Boston, MA 02115, USA
}

\begin{abstract}
How do we find a target item in a visual world filled with distractors? A quarter of a century ago, in her influential 'Feature Integration Theory (FIT)', Treisman proposed a two-stage solution to the problem of visual search: a preattentive stage that could process a limited number of basic features in parallel and an attentive stage that could perform more complex acts of recognition, one object at a time. The theory posed a series of problems. What is the nature of that preattentive stage? How do serial and parallel processes interact? How does a search unfold over time? Recent work has shed new light on these issues.
\end{abstract}

Visual search is one of those things we do all day, every day, from finding milk in the refrigerator to locating our car in the car-park. We pay others to do it at airport security checks and in radiology laboratories and, in the past quarter of a century, we have done a great deal of it in our research laboratories. Laboratory search tasks ask the observer to find and/or identify a target item among some number of distractor items. The core empirical fact that needs explanation is that some search tasks are easy and others are difficult (see Fig. 1). We assume that, if we could successfully describe the rules that govern human search behavior, we would be able to improve performance in critical applied search tasks and to offer suggestions to those trying to build machines that might do our search tasks for us. Visual search is also an experimentally tractable way to study selective attention, and it is increasingly clear that any useful theory of visual perception will require an understanding of the role of attention.

\section{Treisman's feature integration theory}

Visual search and the role of attention in search has been much discussed in recent literature (see [1-5] for reviews). This article will concentrate on several issues growing out of Anne Treisman's seminal Feature Integration Theory (FIT) [6]. It would be a great disservice to many other researchers to label FIT as the sole 'big bang' of the visual search universe. However, it does serve well as an organizing principle for a brief review of some interesting and long-running controversies.

The original FIT proposed that visual search tasks could be dichotomized into 'preattentive' and 'attentive' categories. Preattentive processing was held to occur in parallel across most or all of the visual field in a single step

Corresponding author: Jeremy M. Wolfe (wolfe@search.bwh.harvard.edu). and to be limited to a small set of basic features like color, size, motion, and orientation. Thus, you could preattentively find a red item among green. Operationally, preattentive search for a target defined by a single basic feature would produce reaction times (RTs) independent of the number of items in the display (set size). Thus, the slope of the function relating RT to set size would be near zero. Other tasks, like a search for a randomly oriented ' $T$ ' among 'L's could not be performed preattentively. Attentive processing was presumed to marshal the more extensive perceptual capabilities required to 'bind' features together and discriminate Ts and Ls and so forth. However, it was limited to one item at a time. Thus, search for a T among Ls (or any other attentive search) would need to proceed in a serial manner, from item to item until the target was found or the search was abandoned. This could be seen in RT vs set-size functions that increased at a rate of 20-40 ms/item for target-present trials.

Treisman has modified her original theory and others have proposed variations and alternatives. Still, it is striking how many of the enduring controversies in the field refer back to her framing of the problem. Here we briefly consider three questions.

(1) What is preattentive processing and how is it related to 'early vision' (as defined by visual system physiology) and to attentive vision?



Fig. 1. The core research task in visual search is to explain why some search tasks are easier than others. Finding the target blue-yellow-red 'molecule' is trivial in (a) because of the unique red element. Search is much less efficient in (b) because no unique feature defines the target and because we are particularly bad at search for targets defined by conjunctions of multiple colors. 
(2) Why can't we decide if visual search is a serial or a parallel process?

(3) What is the role of memory in visual search?

The first problem: the nature of preattentive processing Preattentive processing was originally conceived to be quite separate from attentive processing. An entire search task might be described as preattentive. Many current theories hold that preattentive processes guide the deployment of attention to salient items [7-10]. Others suggest that the notion of preattentive processing has outlived its usefulness [11-14]. Still, although it is probably a mistake to imagine an autonomous, physiologically distinct preattentive processor, it is almost tautological to speak of preattentive processing. If there is such a thing as selective attention to some part of the visual field and if there is a time before that portion of the field is selected, then any visual processing at that locus can be defined as preattentive. The interesting question is: "what is the nature of that preattentive processing?'

Twenty years ago, it was tempting to think that the 'basic features' that could be processed preattentively were the same as the features of 'early vision' that were being found to excite neurons in primary or, perhaps, extrastriate visual cortex. Some problems with this hypothesis can be illustrated by considering the simple search for a vertical line.

Finding a vertical among horizontal lines is trivially easy (Fig. 2a). We can do it and cells in primary visual cortex can do it, too. However, the preattentive stage's representation of orientation does not seem to be the same as early vision's representation of that feature. Although cells in the early visual system tend to be sensitive to orientation defined by luminance contrast, a vertical target in visual search can be defined by many different surface properties: Color, motion, texture (Fig. 2b) [15] - even a vertical group of smaller horizontally oriented elements can form a vertical target [16]. Moreover, preattentive processes seem unable to use the full capabilities of early visual processing of orientation. With attention, we can easily discriminate between a vertical ( $0 \mathrm{deg})$ line and one tilted a degree or two to either side. Early cortical cells possess the information to perform these discriminations [17].
As illustrated in 2c, the preattentive just noticeable difference' in visual search is much cruder [18]. Search for the vertical target among items tilted $5 \mathrm{deg}$ to the right is difficult. The situation is similar for other dimensions (e.g. color [19]). In fact, it may be that preattentive processes only use information about the categorical status of items. In orientation, that means that it is only easy to find a target if it is uniquely 'steep', 'shallow', 'tilted left' or 'right [20,21]. Thus, vertical is hard to find among $\pm 20 \mathrm{deg}$ (Fig. $2 \mathrm{~d}$ ) because all of the items are 'steep' and the target is tilted neither left nor right.

There is a paradox here. Preattentive processes seem to have properties associated with later stages of visual cortical processing [22]. Attentive processing seems to have access to the information held by cells in early visual cortex. Yet, by definition, 'preattentive' is what happens before an object is attended. The paradox can be resolved by recalling that the visual pathway is not a one-way street. Thus, salvation may come from feedback or 're-entrant' [23] pathways in the visual system (see also Box 1). Preattentive processing may represent a fast but relatively high-level abstraction of the visual scene. On the basis of that abstraction, attention selects specific objects for further processing. Part of that further processing involves reaching back to the earlier stages of processing for the fine detail that was not used by the preattentive processes (see [12] and the reverse hierarchy theory of Hochstein and Ahissar [24]). (see Fig. 3)

\section{The second problem: is search serial or parallel?}

In FIT, the second, attentive stage of processing is serial in nature. That is, attention is deployed to one item (or, perhaps, to one group of items [25]) at a time. Under FIT's assumption of a serial, self-terminating search, the time for each covert deployment of attention can be estimated from the slope of the RT vs set-size function because the slope is linearly related to the added cost of each added item in the display. Estimates of 25-60 ms are fairly standard [26]. (Overt deployments of the eyes are slower: $150-200 \mathrm{~ms}$ per saccade). These pose a problem if one supposes that this is an estimate of the time required to process each (a)

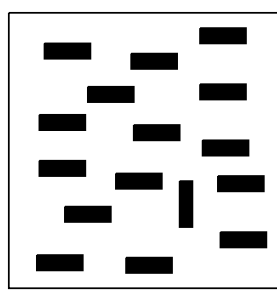

(b)

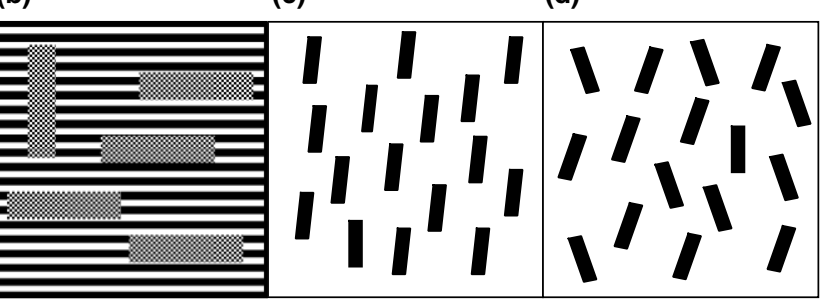

Fig. 2. Search for vertical ( $0 \mathrm{deg}$ ) among horizontal bars is easy (a), even if the items are defined by properties other than luminance contrast: texture (b), motion, depth, etc. Search for 0 deg among 5 deg tilted bars is hard (c), even though perceptual orientation-discrimination thresholds are much lower than 5 deg and cortical cells are sensitive to differences in orientation of less than 5 deg. Search for 0 deg among \pm 20 deg is hard (d), even though search for 0 deg among +20 would be easy. 


\section{Box 1. Using preattentive information: top-down and bottom-up guidance}

The Guided Search (GS) model [a,b] was a response to a problem with the original Feature Integration Theory (FIT) [c]. FIT had proposed a division between parallel, preattentive searches and serial, attentive searches. This dichotomy does not, however, appear in the data. In particular, FIT proposed that searches for conjunctions of two (or more) features should have been serial. However, they often proved to be much more efficient than serial search would predict [d], sometimes as efficient as presumed preattentive searches [e]. GS kept the basic preattentive/attentive structure but proposed that preattentive processes could guide the deployment of the serial, attentive stage. In GS and a number of related models, there are several ways for preattentive information to guide the deployment of attention. There is bottom-up, stimulus-driven guidance to salient items $[f, g]$. The attraction of attention to a salient item might not be mandatory capture but may be contingent on task demands $[\mathrm{h}, \mathrm{i}]$. Another mechanism, top-down guidance, is based on the needs of the searcher [j]. Top-down control can be a response to explicit task demands ('look for red vertical') or it can be an implicit change in guidance, for example, based on the previous identity of targets (known as 'priming of pop-out') [k].

Like FIT, GS was faced with the paradox of a preattentive stage that seemed to have thrown away information that would be needed by the subsequent attentive stage. This is well-accounted for by adopting the feedback/re-entrant ideas sketched in Fig. I (and Fig. 3 in main text).

\section{References}

a Wolfe, J.M. et al. (1989) Guided search: an alternative to the feature integration model for visual search. J. Exp. Psychol. Hum. Percept. Perform. 15, 419-433 b Wolfe, J.M. (1994) Guided Search 2.0: a revised model of visual search. Psychonomic Bull. Rev. 1, 202-238

c Treisman, A. and Gelade, G. (1980) A feature-integration theory of attention. Cogn. Psychol. 12, 97-136

d Nakayama, K. and Silverman, G.H. (1986) Serial and parallel processing of visual feature conjunctions. Nature 320, 264-265

e Theeuwes, J. and Kooi, J.L. (1994) Parallel search for a conjunction of shape and contrast polarity. Vision Res. 34, 3013-3016

f Nothdurft, H.-C. (2000) Salience from feature contrast: additivity across dimensions. Vision Res. 40, 1183-1201

g Li, Z. (2002) A salience map in primary visual cortex. Trends Cogn. Sci. 6, 9-16

h Folk, C.L. et al. (1994) The structure of attentional control: contingent attentional capture by apparent motion, abrupt onset, and color. J. Exp. Psychol. Hum. Percept. Perform. 20, 317-329

i Yantis, S. and Egeth, H.E. (1999) On the distinction between visual salience and stimulus-driven attentional capture. J. Exp. Psychol. Hum. Percept. Perform. 25, 661-676

j Hodsoll, J. and Humphreys, G.W. (2001) Driving attention with the top down: the relative contribution of target templates to the linear separability effect in the size dimension. Percept. Psychophys. 63, 918-926

$\mathrm{k}$ Kristjansson, A. et al. (2002) The role of priming in conjunctive visual search. Cognition 85, 37-52

1 Treisman, A. (1998) Feature binding, attention and object perception. Philos. Trans. R. Soc. Lond. B Biol. Sci. 353, $1295-1306$ (a) Pre-attentive processing

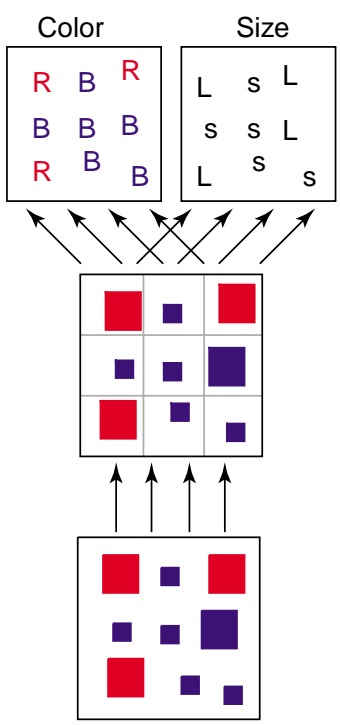

Feed-forward (b) Feedback for selection

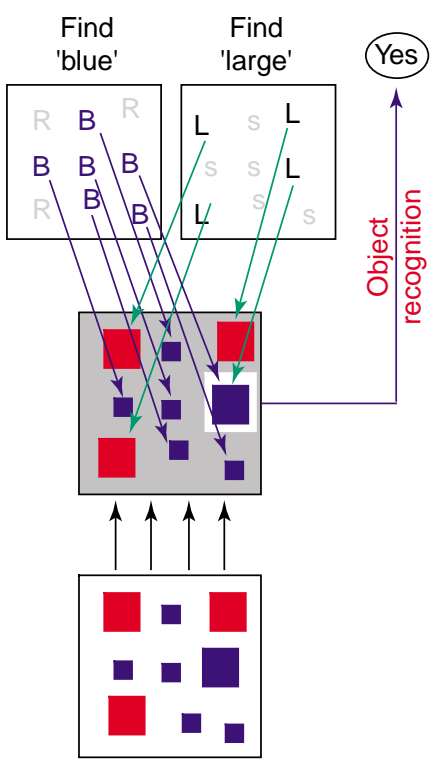

$\overline{T R E N D S ~ i n ~ C o g n i t i v e ~ S c i e n c e s ~}$

Fig. I. Guided search for a conjunction. When observers search for a conjunction of two features (e.g. the large, blue target in the stimulus shown), bottom-up salience information is relatively useless. Nevertheless, visual search remains efficient. Models like Guided Search [a,b] propose that feed-forward, pre-attentive processing can establish the locations of objects with properties like 'blue' and 'large' (a) even if no feed-forward process can be used to recognize a large-blue item. (b) Using top-down guidance, the preattentive information can then be fed back in order to select items that are both blue and large. Attending to such an item can confirm that it is, indeed, a large blue target, perhaps by 'binding' the features into a single object representation [I]. 


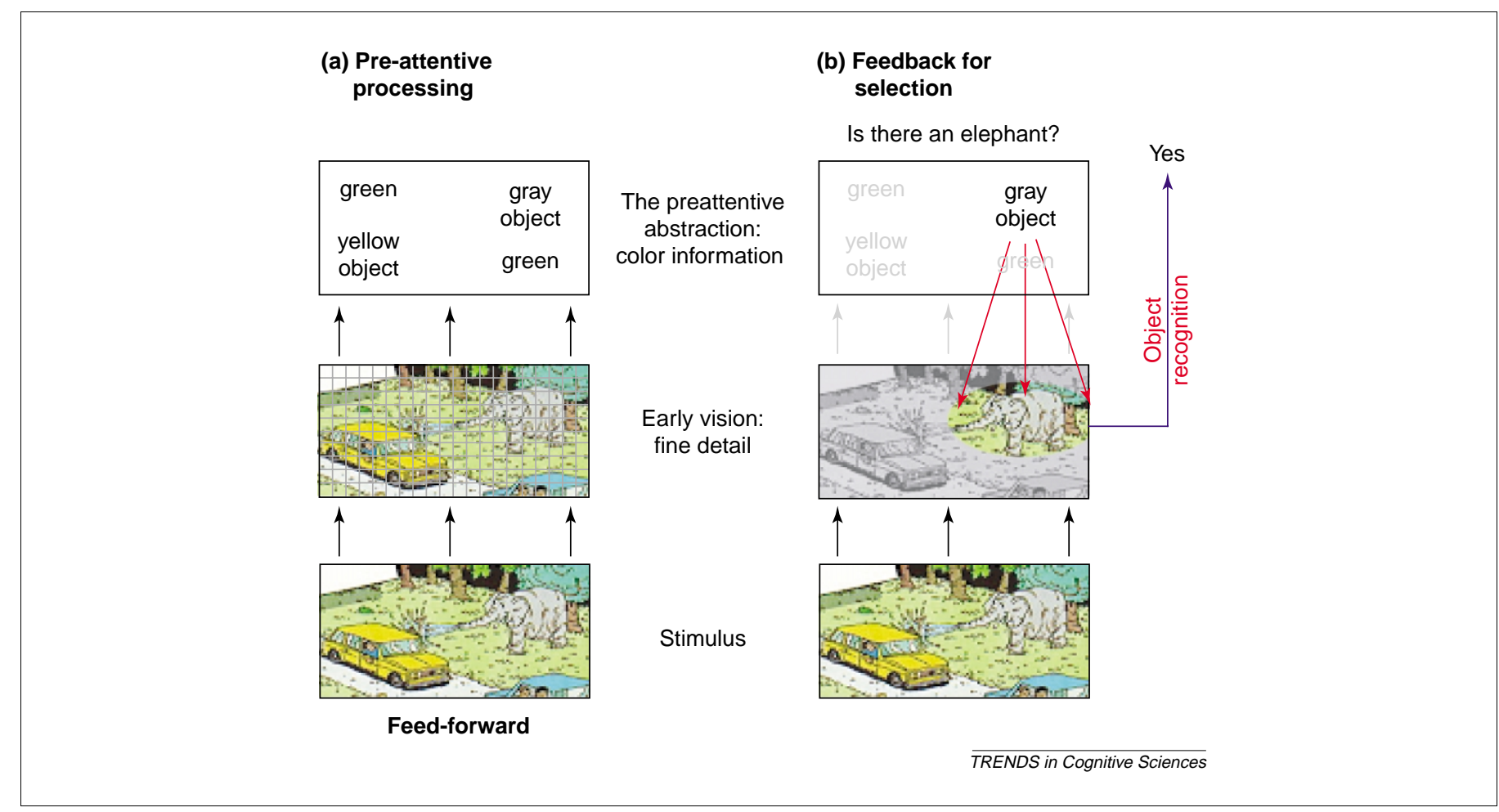

Fig. 3. The 'pre-attentive processing' used to guide attention appears to be a coarse abstraction of the visual input. However, subsequent, attentive processing has access to the fine detail available in 'early vision' (middle layers above). Clearly, the fine detail was not lost in the creation of the preattentive representation. How is this possible? Preattentive processing may be the product of fast, feed-forward processing (a). Much visual information is extracted in parallel by early vision and then coarsely coded into a preattentive representation of a few basic features. Little information about the meaning of the scene is available (although see Refs [27,59]). (b) Feedback based on that coarse, high-level, preattentive information can be used to select part of the stimulus/early vision representation for the attentive processing required for object recognition. Inspired by the reverse hierarchy theory of Ahissar and Hochstein [24]. (Illustration from Where's Waldo? (C) 1987, 1997 Martin Handford. Reproduced by permission of Walker Books Ltd, London. Published by Candlewick Press Inc., Cambridge, MA, USA.)

item in visual search. No credible mechanism of object recognition works that fast (see for example [27,28]). Moreover, estimates of the attentional 'dwell time' derived from paradigms like the 'attentional blink' [29,30] are in the range of $200-500 \mathrm{~ms}$.

One solution to this problem has been to propose that search does not have a serial component. Perhaps object recognition is accomplished by a parallel process that is capable of processing many items at once. Different types of parallel process can be devised that will produce the same patterns of data that led Treisman and others to propose a serial selfterminating search [13].

One problem with parallel accounts of search is that there are many situations in which it is clear that the visual system uses selective attention to avoid processing all items at once [31]. If an observer is given a cue that the target is likely to be at location $\mathrm{X}$ and not at $\mathrm{Y}$, the observer attends to $\mathrm{X}$ [32]. It can be shown that detection of small probe stimuli is enhanced on and immediately around the attended object [33]. One can even mimic a set size variation by simply telling observers which items to attend to on any given trial [34]. It may be that spatially selective attention serves a useful role in collecting features from just one object at a time and delivering those features to later object recognition processes [35]. This might help avoid 'illusory' conjunctions between the features of different objects [36].
Perhaps the serial/parallel debate in visual search has eluded resolution for more than two decades because the same search mechanism can look either serial or parallel, depending on our experimental vantage point. A carwash provides a useful metaphor (Fig. 4). Cars enter the carwash in series. However, at any one moment, several cars are being washed in parallel. The slope of the RT vs set-size function does not tell us how long it takes to wash each car. It merely describes the rate at which cars pass through the system. Thus, visual search 'cars' might enter the carwash every $50 \mathrm{~ms}$ - producing a $50 \mathrm{~ms} /$ item slope - but still take $300 \mathrm{~ms}$ to get washed - producing a $300 \mathrm{~ms}$ 'dwell time'. Design an experiment one way, and the carwash will look 'serial'. Look at it another way and it will appear to be a parallel processor.

A carwash is a metaphor, not a model. It is a metaphor for a large class of possible models that are hybrids of parallel and serial processes. The idea is not new, even in visual search [37]. In computer science, 'pipeline' processes are an example of this sort of model. Continuing in metaphorical terms, one might wonder if all cars take the same time to wash. Could car A enter after car B but leave before B? Could more than one car enter at a time? Is the speed of washing dependent on the number of cars in the carwash? Is it possible to wash a car twice at the same time. This last possibility would be nonsense in a physical carwash. However, in search, if items are selected every $50 \mathrm{~ms}$ and are then processed for 300 , it becomes logically possible for an item to be selected two or more times within 
(a) A serial carwash

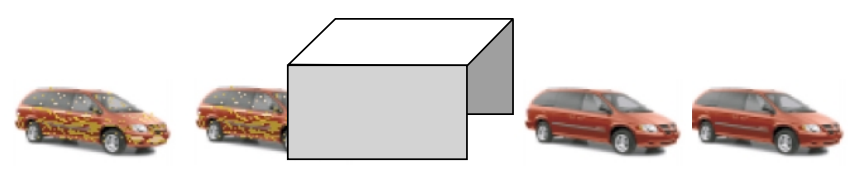

(b) A parallel carwash

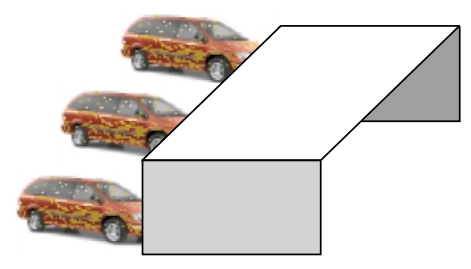

(c) A hybrid 'pipeline' carwash

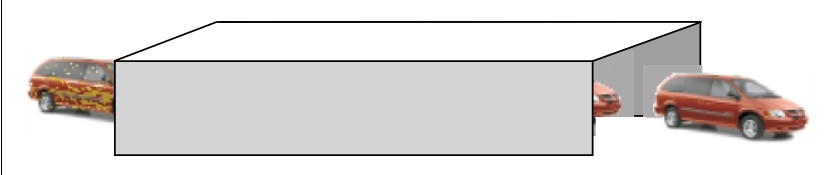

$\overline{\text { TRENDS in Cognitive Sciences }}$

Fig. 4. A classic debate in the visual search literature has pitted proponents of 'serial' models against proponents of 'parallel' models. A carwash can be used as a metaphor. (a) In a serial carwash, cars are washed one at a time. (b) In a parallel carwash, all the cars are washed in a single step. (c) Perhaps the true situation is a hybrid - rather like a real carwash, in fact. Cars enter one at a time, in series. However, multiple cars are in the wash at the same time, giving the process a parallel aspect as well.

the same $300 \mathrm{~ms}$ period. What would a second act of selection do to the processing of an item already in the carwash?

The third problem: the role of memory in visual search The notion of washing the same car twice points to the last controversy in this brief survey. Do observers keep track of the course of a visual search? Models with a serial components, like FIT and Guided Search (GS), have usually assumed that items, once rejected as distractors, are not revisited in the course of a search. The idea, in the original FIT, that target absent trials should have slopes that are twice as steep as the target-present slopes arises from the assumption that observers examine all items once and only once on absent trials. Attending to a distractor only once seems like a very sensible thing to do.

The most frequently proposed mechanism to avoid revisiting rejected distractors ('sampling without replacement') is known as 'inhibition of return' (IOR) [32]. In a typical IOR experiment, an observer's attention is attracted to one location and then back to fixation. When asked to respond to a subsequent stimulus at the same or at a different location, observers are slower to respond at the previously attended location - as if they were inhibited in their effort to get attention back to that object. Klein [38] found IOR at distractor locations in a visual search task and argued that IOR made visual search more efficient. There has been some controversy about this finding [39] but in the last few years it has become fairly clear that IOR can be seen in search paradigms [40,41]. Still, the role of IOR in search is not entirely clear. IOR typically takes $\sim 200-300 \mathrm{~ms}$ to develop. At search rates of $25-50 \mathrm{~ms} / \mathrm{item}$, small set size searches would be over before IOR could be of any use. Moreover, IOR seems to be present only at the last 4-6 attended items $[42,43]$. Thus, with larger set sizes, IOR would have worn off before the entire set could have been sampled without replacement. IOR might be able to keep attention from going back to the last few items. This could be a way to prevent perseveration on one salient item even if it did not successfully mark all attended items.

Problems with IOR do not preclude the possibility that some mechanism tracks rejected distractors during search. To examine the role of memory for rejected distractors in visual search, we tried to disrupt IOR or equivalent mechanisms. [44]. We compared a standard 'serial' search task with a dynamic version in which every item was randomly repositioned every $100 \mathrm{~ms}$ or so (Fig. 5). Clearly, observers could not keep track of the course of search in this dynamic case. Nevertheless, slopes of the RT vs set-size functions were about the same in static and dynamic conditions. Given that observers could not keep track of rejected distractors in the dynamic conditions, our results suggested that they were not keeping track in the static condition either. We argued that 'visual search has no memory' for rejected distractors [44]. This has been a controversial claim (e.g. [45]) and, even though we have replicated [46] and extended [47] the original finding, the bold claim of complete amnesia is probably too strong.

First of all, no one ever doubted that there was memory for the location of detected targets in search [48] and observers can learn where targets are likely to be in familiar displays [49]. Within a search, there is a form of memory in strategically planned searches. Imagine reading a page in search of a word. You would know where you have been although these searches proceed at a rate much slower than that of standard

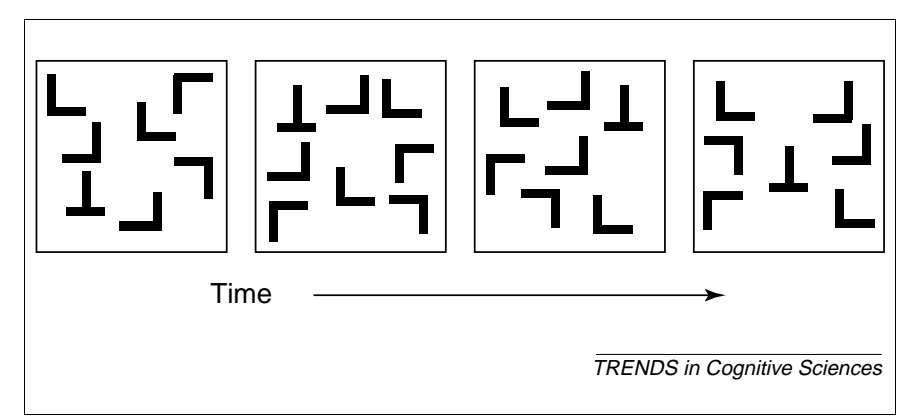

Fig. 5. Models of search have tended to assume that the searcher can keep track of rejected distractors so as not to attend to them again. In a 'dynamic search' task, the target (here, a ' $T$ ' shape among ' $L$ 's) is either present or absent for the whole trial but all items are repositioned every $100 \mathrm{~ms}$ or so. Observers cannot keep track of the rejected distractors in a dynamic search; nevertheless, search efficiency, as measured by the slope of the reaction-time vs set-size function, tends to be the same in dynamic and static search. 
laboratory search [50]. Most importantly for the present discussion, there seems to be some memory for deployments of the eyes [51-53]. Interestingly, the interval between voluntary eye movements is similar to the time required for the development of IOR. This may point the way to a compromise view of the role of memory in search. The Horowitz and Wolfe experiments confirm what should have been clear from the IOR data: observers are not searching without replacement from visual displays. Models that propose perfect memory will have to be modified. At the same time, the IOR and the eye movement data indicate that observers are not completely amnesic about the progress of search. Processes, perhaps related to eye movement control, may exist to prevent perseveration on salient distractors. The details of a partial memory model of search remain to be worked out (see [54]), but it would be reasonable to propose that visual search has, at best, a rather modest memory.

\section{Where do we go next?}

To summarize, a good case can be made for a view of visual search in which relatively crude, categorical preattentive processes guide the serial deployment of attention from object to object or, perhaps, from one group of objects to the next. Selective attention allows a manageable subset of the information in early vision to be delivered to limited-capacity object recognition mechanisms. Attention may deliver items every $25-50 \mathrm{~ms}$ but it takes much longer for a bundle of features to be bound into a recognizable object. Thus, several objects may be working their way toward recognition at the same time, giving search simultaneous serial and parallel qualities. In selecting objects, the search mechanism does not completely ignore the prior history of search, but neither does it keep any reliable record of rejected distractors.

This is, by no means, the only way to conceptualize visual search. For example, one could propose that the only serial aspect to search is provided by eye movements, and that processing is parallel within a 'useful' or 'functional field of view' surrounding fixation [55]. If we don't know 'The Truth' yet, how can we move forward in this area? One route is to move beyond studies of mean reaction time (and accuracy). Mean RTs are extremely useful but there are many models that produce roughly the correct pattern of mean RTs. Can these models predict other aspects of the data RT distributions, for example? To find out, we will need datasets that are richer than those currently available. For example, we have posted an extensive set that can be used to model RT distributions [56].

This article is too short to do justice to some of the other methods for going beyond simple RT data. For example, McElree and Carrasco's [57] work on speed-accuracy tradeoffs or Lu and Dosher's [58] modeling of the effects of external noise.

\section{Why should we care?}

When Anne Treisman started her work, attention was a relatively small topic in psychology. It had been important in the 19th century but had fallen out of favor. With the rise of cognitive science, it has become ever clearer that our mental life and behavior are critically dependent on selection mechanisms that allow us to choose some things at the expense of others. The study of visual search does not exhaust the interesting questions about attention. However, although we might be interested in our ability to direct attention to TICS rather than to lunch, it is currently more tractable to study our ability to attend to one visual stimulus rather than another. If we understand visual search, perhaps we can make progress in other attentional realms. Moreover, if we understood search in the laboratory, we might be able to provide useful advice those who create artificial but critical search tasks. From airport security screening to the design of computer interfaces to training of radiologists; our health and safety rely, in part, on successful search.

\section{References}

1 Kinchla, R.A. (1992) Attention. Annu. Rev. Psychol. 43, 711-742

2 Wolfe, J.M. (1998) Visual search. In Attention (Pashler, H., ed.), pp. 13-74, Psychology Press Ltd

3 Sanders, A.F. and Donk, M. (1996) Visual search. Handbook of Perception and Action (Vol. 3) (Neumann, O., Sanders, A.F. eds), pp. 43-77, Academic Press

4 Chun, M.M. and Wolfe, J.M. (2001) Visual Attention. In Blackwell's Handbook of Perception (Vol. Ch 9) (Goldstein, E.B., ed.), pp. $272-310$

5 Egeth, H.E. and Yantis, S. (1997) Visual Attention: Control, representation, and time course. Annu. Rev. Psychol. 48, 269-297

6 Treisman, A. and Gelade, G. (1980) A feature-integration theory of attention. Cogn. Psychol. 12, 97-136

7 Wolfe, J.M. et al. (1989) Guided Search: an alternative to the Feature Integration model for visual search. J. Exp. Psychol. Hum. Percept. Perform. 15, 419-433

8 Nothdurft, H-C. (2000) Salience from feature contrast: additivity across dimensions. Vision Res. 40, 1183-1201

9 Itti, L. and Koch, C. (2000) A saliency-based search mechanism for overt and covert shifts of visual attention. Vision Res. 40, $1489-1506$

$10 \mathrm{Li}, \mathrm{Z}$. (2002) A salience map in primary visual cortex. Trends Cogn. Sci. $6,9-16$

11 Nakayama, K. and Joseph, J.S. (1998) Attention, pattern recognition and popout in visual search. In The Attentive Brain (Parasuraman, R., ed.), pp. 279-298, MIT Press

12 DiLollo, V. et al. (2001) The preattentive emperor has no clothes: a dynamic redressing. J. Exp. Psychol. Gen. 130, 479-492

13 Palmer, J. et al. (2000) The psychophysics of visual search. Vision Res. $40,1227-1268$

14 Verghese, P. (2001) Visual search and attention: A signal detection approach. Neuron $31,523-535$

15 Cavanagh, P. et al. (1990) Effect of surface medium on visual search for orientation and size features. J. Exp. Psychol. Hum. Percept. Perform. $16,479-492$

16 Bravo, M. and Blake, R. (1990) Preattentive vision and perceptual groups. Perception 19, 515-522

17 Regan, D. and Beverly, K.I. (1985) Postadaptation orientation discrimination. J. Opt. Soc. Am. A 2, 147-155

18 Foster, D.H. and Westland, S. (1995) Orientation contrast vs orientation in line-target detection. Vision Res. 35, 733-738

19 Nagy, A.L. and Sanchez, R.R. (1990) Critical color differences determined with a visual search task. J. Opt. Soc. Am. A 7, $1209-1217$

20 Foster, D.H. and Ward, P.A. (1991) Horizontal-vertical filters in early vision predict anomalous line-orientation frequencies. Proc. $R$. Soc. Lond. B Biol. Sci. 243, 83-86

21 Wolfe, J.M. et al. (1992) The role of categorization in visual search for orientation. J. Exp. Psychol. Hum. Percept. Perform. 18, 34-49 
22 Laack, K.A. and Olzak, L.A. (2002) Orientation-selective summing mechanisms revealed in visual search. Vision Res. 42, 1871-1877

23 Di Lollo, V. et al. (2000) Competition for consciousness among visual events: the psychophysics of reentrant visual processes. J. Exp. Psychol. Gen. 129, 481-507

24 Ahissar, M. and Hochstein, S. (1997) Task difficulty and visual hierarchy: Counter-streams in sensory processing and perceptual learning. Nature 387, 401-406

25 Treisman, A. (1982) Perceptual grouping and attention in visual search for features and for objects. J. Exp. Psychol. Hum. Percept. Perform. 8, 194-214

26 Wolfe, J.M. (1998) What do 1,000,000 trials tell us about visual search? Psychol. Sci. 9, 33-39

27 VanRullen, R. and Thorpe, S.J. (2001) Is it a bird? Is it a plane? Ultrarapid visual categorisation of natural and artifactual objects. Perception 30, 655-668

28 Thorpe, S. et al. (1996) Speed of processing in the human visual system. Nature $381,520-552$

29 Raymond, J.E. et al. (1992) Temporary suppression of visual processing in an RSVP task: an attentional blink? J. Exp. Psychol. Hum. Percept. Perform. 18, 849-860

30 Duncan, J. et al. (1994) Direct measurement of attention dwell time in human vision. Nature 369, 313-314

31 Cave, K.R. and Bichot, N.P. (1999) Visuo-spatial attention: Beyond a spotlight model. Psychon. Bull. Rev. 6, 204-223

32 Posner, M.I. and Cohen, Y. (1984) Components of attention. Attention and Performance $X$ (Bouma, H., Bouwhuis, D.G. eds), pp. 55-66, Erlbaum

33 Kim, M. and Cave, K.R. (1995) Spatial attention in visual search for features and feature conjunctions. Psychol. Sci. 6, 376-380

34 Palmer, J. et al. (1993) Measuring the effect of attention on simple visual search. J. Exp. Psychol. Hum. Percept. Perform. 19, $108-130$

35 Wolfe, J.M. and Cave, K.R. (1999) The psychophysical evidence for a binding problem in human vision. Neuron 24, 11-17

36 Treisman, A.M. and Schmidt, H. (1982) Illusory conjunctions in the perception of objects. Cogn. Psychol. 14, 107-141

37 Harris, J.R. et al. (1979) Visual search in multicharacter arrays with and without gaps. Percept. Psychophys. 26, 69-84

38 Klein, R. (1988) Inhibitory tagging system facilitates visual search. Nature 334, 430-431

39 Wolfe, J.M. and Pokorny, C.W. (1990) Inhibitory tagging in visual search: A failure to replicate. Percept. Psychophys. 48, 357-362

40 Takeda, Y. and Yagi, A. (2000) Inhibitory tagging in visual search can be found if search stimuli remain visible. Percept. Psychophys. 62 , 927-934

41 Muller, H. and von Muhlenen, A. (2000) Probing distractor inhibition in visual search: Inhibition of return. J. Exp. Psychol. Hum. Percept. Perform. 26, 1591-1605

42 Snyder, J.J. and Kingstone, A. (2000) Inhibition of return and visual search: how many separate loci are inhibited? Percept. Psychophys. 62 , $452-458$

43 Danziger, S. et al. (1998) Inhibition of return to successively stimulated locations in a sequential visual search paradigm. J. Exp. Psychol. Hum. Percept. Perform. 24, 1467-1475

44 Horowitz, T.S. and Wolfe, J.M. (1998) Visual search has no memory. Nature $394,575-577$

45 Kristjansson, A. (2000) In search of rememberance: Evidence for memory in visual search. Psychol. Sci. 11, 328-332

46 Horowitz, T.S. and Wolfe, J.M. Memory for rejected distractors in visual search? Visual Cognition (in press)

47 Horowitz, T.S. and Wolfe, J.M. (2001) Search for multiple targets: Remember the targets, forget the search. Percept. Psychophys. 63, $272-285$

48 Gibson, B.S. et al. (2000) Searching for one versus two identical targets: when visual search has a memory. Psychol. Sci. 11 $324-327$

49 Chun, M. and Jiang, Y. (1998) Contextual cuing: Implicit learning and memory of visual context guides spatial attention. Cogn. Psychol. 36, 28-71

50 Wolfe, J. et al. (2000) Attention is fast but volition is slow. Nature 406 691

51 Klein, R.M. and MacInnes, W.J. (1999) Inhibition of return is a foraging facilitator in visual search. Psychol. Sci. 10, 346-352

52 Gilchrist, I.D. et al. (2000) Memory in visual search: an eye movement study. Perception ECVP abstract, S86

53 Peterson, M.S. et al. (2000) Visual search has memory. Psychol. Sci. 12 , 287-292

54 Arani, T. et al. (1984) A variable-memory model of visual search. Hum. Factors 26, 631-639

55 Sanders, A.F. and Houtmans, M.J.M. (1985) Perceptual modes in the functional visual field. Acta Psychol. (Amst.) 58, 251-261

56 http://search.bwh.harvard.edu/links\%20from\%20homepage/ Data\%20set/Datasets.html

57 McElree, B. and Carrasco, M. (1999) The temporal dynamics of visual search: evidence for parallel processing in feature and conjunction searches. J. Exp. Psychol. Hum. Percept. Perform. 25, 1517-1539

$58 \mathrm{Lu}$, Z-L. and Dosher, B.A. (1998) External noise distinguishes attention mechanisms. Vision Res. 38, 1183-1198

59 Oliva, A. and Torralba, A. (2001) Modeling the shape of the scene: a holistic representation of the spatial envelope. Int. J. Comput. Vis. 42, $145-175$

\section{The BioMedNet Magazine}

The new online-only BioMedNet Magazine contains a range of topical articles currently available in Current Opinion and Trends journals, and offers the latest information and observations of direct and vital interest to researchers.

You can elect to receive the BioMedNet Magazine delivered directly to your e-mail address, for a regular and convenient survey of what's happening outside your lab, your department, or your speciality.

Issue-by-issue, the BioMedNet Magazine provides an array of some of the finest material available on BioMedNet dealing with matters of daily importance: careers, funding policies, current controversy and changing regulations in the practice of research. 